\title{
Computed Tomography of Cortical Desmoid
}

\author{
D.R. Pennes, M.D., E.M. Braunstein, M.D., and G.M. Glazer, M.D. \\ Department of Radiology, University of Michigan Medical Center, Ann Arbor, Michigan, USA
}

\begin{abstract}
The diagnosis of cortical desmoid in the typical case is easily established using conventional radiography. In atypical cases, the use of computed tomography $(\mathrm{CT})$ may be helpful in diagnosis. We report the use of computed tomography in two patients with cortical desmoids. In one case plain radiographic findings were not diagnostic and the use of CT obviated biopsy. The finding of a desmoid in an atypical location, the insertion site of the medial head of the gastrocnemius tendon, is discussed.
\end{abstract}

Address reprint requests to: David R. Pennes, M.D., Department of Radiology, Box 013 University of Michigan Hospitals, Ann Arbor, MI 48109, USA
Key words: Tomography, X-ray computed - Bone, diagnosis - Ligament, stress, mechanical - Tendon injuries

Cortical irregularities of the distal femur are known by a variety of terms including cortical desmoid [6], periosteal desmoid [7], parosteal (juxtacortical) desmoid [8], or avulsive cortical irregularity [4]. These lesions are believed to result from chronic stress, and they occur on the posteromedial surface of the distal femoral metaphysis at the attachment of the adductor aponeurosis to the me-
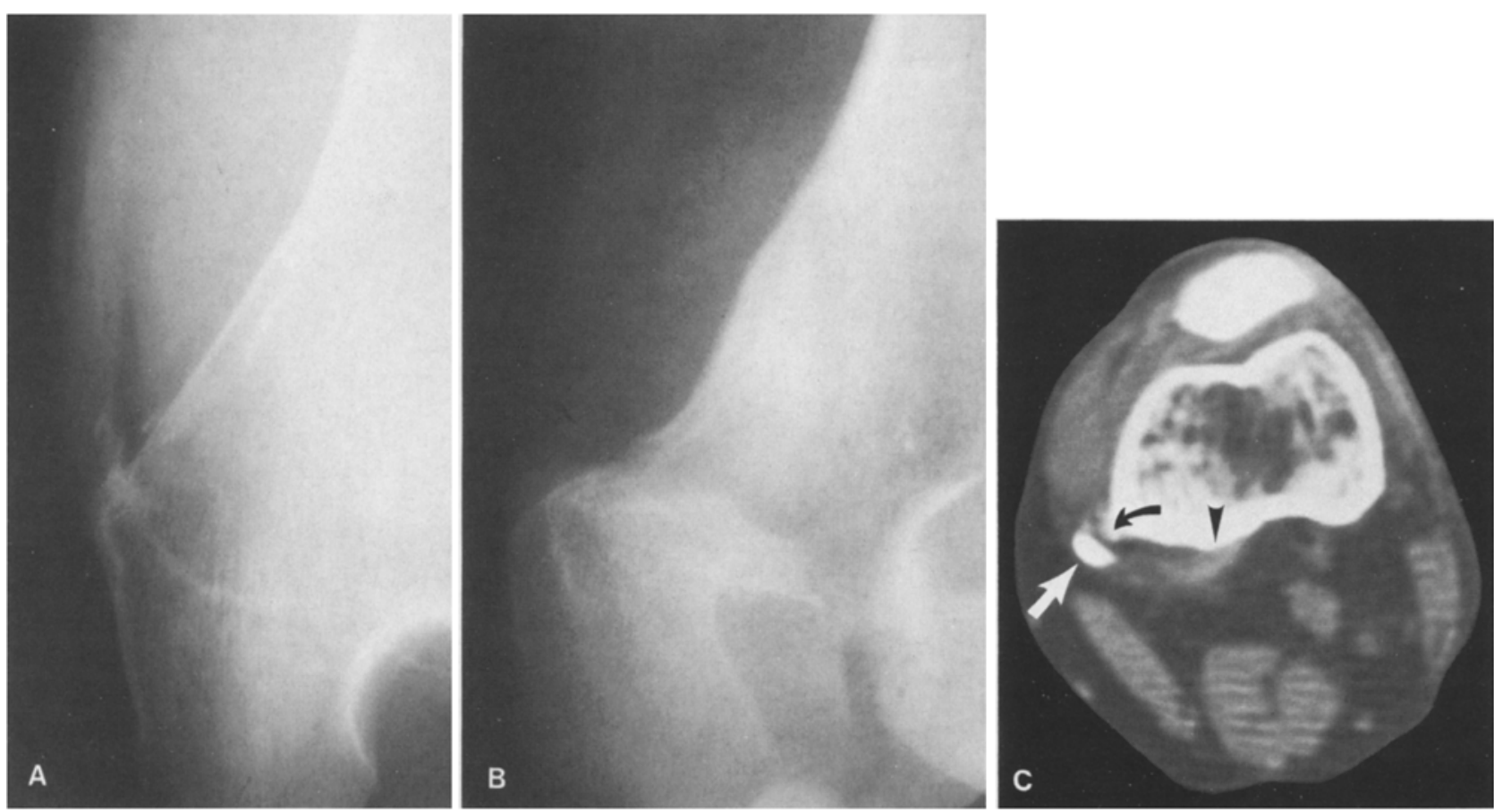

Fig. 1 A-C. Case 1. A Notch view of the left medial femoral condyle shows a cortical avulsion fracture. B Oblique view confirms avulsion fracture. C CT scan shows avulsed fragment of bone (arrow) adjacent to the adductor tubercle (curved arrow). Insertion of the medial head of gastrocnemius muscle (arrowhead) 

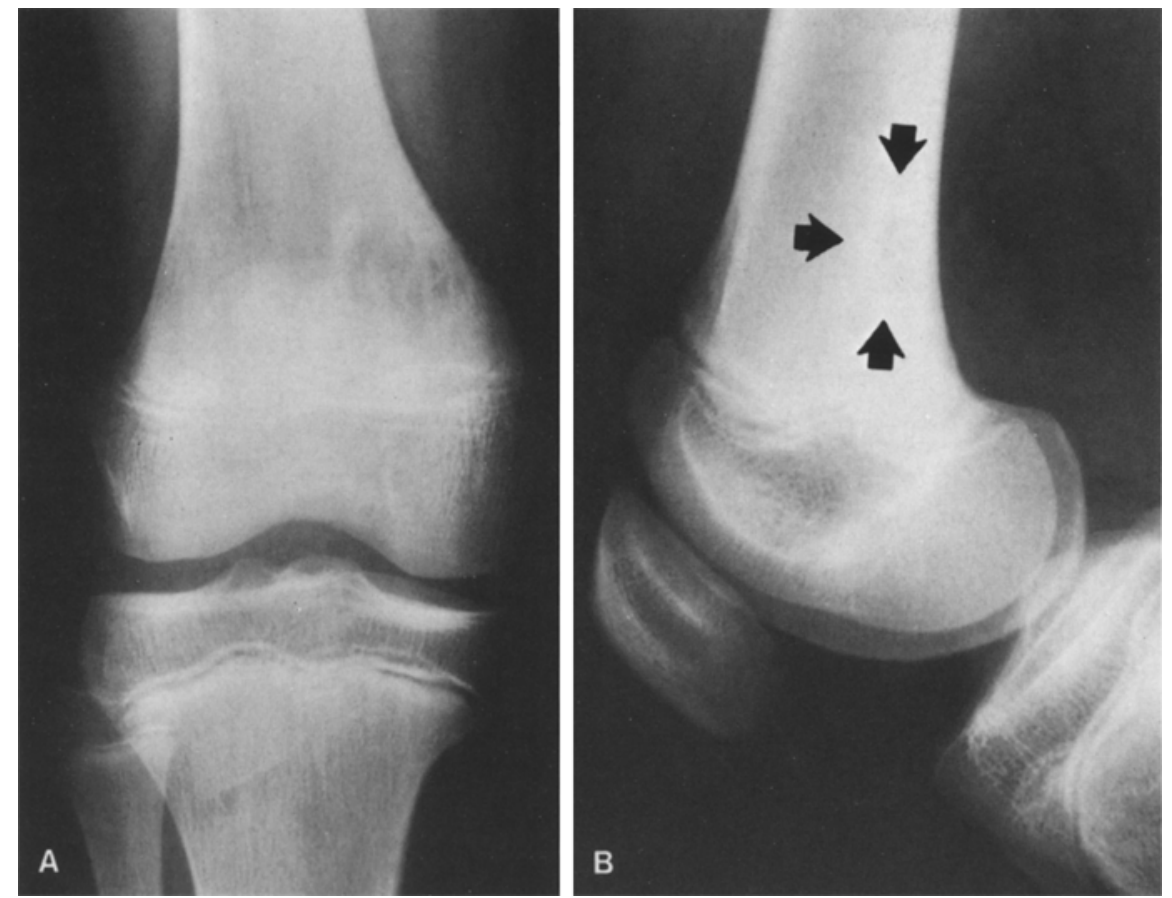

Fig. 2 A-D. Case 2

A Anteroposterior view of the right knee demonstrates a lytic lesion with sclerosis and periosteal reaction

B Lateral view. Cortical thickening posteriorly (arrows) C CT scan demonstrates focal cortical thickening and minimal fragmentation corresponding to the lytic area of Fig. $1 \mathrm{~A}$ at the insertion of the medial head of the gastrocnemius tendon (arrows). Identical finding is present on the left

D CT scan of the right leg at a slightly caudal level shows the adductor magnus tendon (arrow) adjacent and medial to the insertion of the tendon (curved arrow) of the medial head of the gastrocnemius muscle (M)
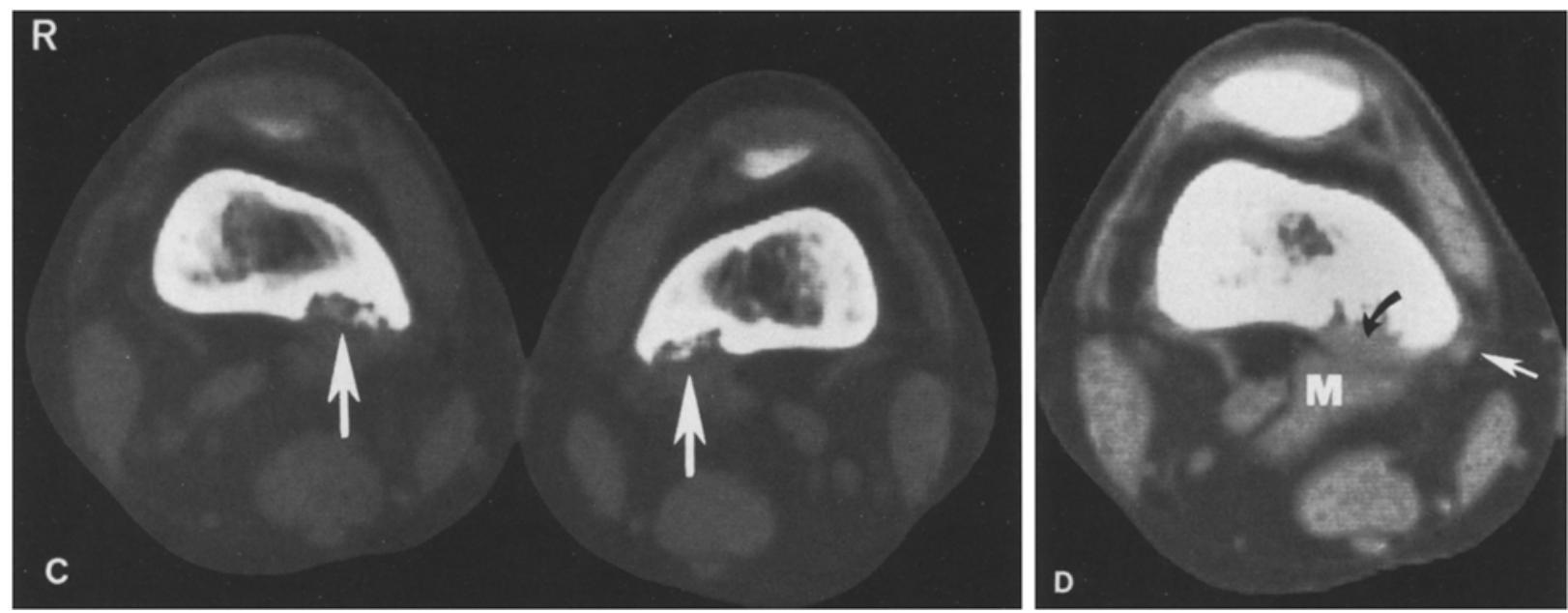

dial supracondylar ridge [2]. The usual appearance consists of a radiolucent cortical defect with adjacent sclerosis and periostitis in the characteristic location [5]. Since most of the lesions have a typical appearance, appropriate anteroposterior, later$\mathrm{al}$, and oblique radiographs are generally sufficient for accurate diagnosis. Cortical desmoids may occasionally have atypical appearances suggesting malignancy when an associated soft tissue mass or excessive periosteal proliferation are present [1, $2,5]$.

We report the use of computed tomography (CT) in two patients with cortical desmoids. In one case plain radiographic findings were not diagnostic and the use of CT obviated biopsy. The finding of a desmoid in an atypical location, the insertion site of the medial head of the gastrocnemius tendon, is discussed.

\section{Case Reports}

Case 1

A 57-year-old male with a remote history of knee trauma was evaluated for chronic pain. Radiographs (Fig. 1A and B) demonstrated a cortical avulsion in the location of the adductor magnus tendon insertion. A CT scan of this region (Fig. 1 C) confirmed the conventional radiographic findings.

\section{Case 2}

A 13-year-old female presented with persistent right knee pain of one year duration. Radiographs demonstrated a lytic lesion with a sclerotic margin and periosteal reaction in the distal femur (Fig. 2A and B). Differential diagnosis included osteo- 
myelitis or less likely osteoblastoma. A CT scan was requested prior to biopsy to exclude an associated soft tissue mass (Fig. 2C). The CT scan showed bilaterally symmetrical, sclerotic, cortical abnormality at the insertion site of the medial head of the gastrocnemius tendon. Because of the CT findings, biopsy was not performed. Radiographs of the left knee following the CT scan demonstrated a similar lesion. Follow-up questioning of the patient revealed no history of unusually strenuous physical activity.

\section{Discussion}

Cortical irregularities of the distal femur vary in severity from a minimal degree of indistinctness of the medial metaphysis to ragged spiculation with an associated mass simulating malignancy $[1$, $2,5,8]$. In most cases the diagnosis of cortical desmoid can be made with certainty based on the conventional radiographic appearance. In cases with equivocal radiographic findings, a history of unusually strenuous physical activity may point toward the correct diagnosis [8]. In unusual instances, when the diagnosis is not evident from conventional films or history as in Case 2, CT may prove useful and make biopsy unnecessary. This is important since biopsy of such lesions can suggest malignancy $[4,6-8]$, resulting in unnecessary amputation [7]. Cortical irregularities of the medial distal femoral metaphysis have been reported to occur in $11.5 \%$ of male and $3.6 \%$ of female children between the ages of 3 and 17 years [11]. Although cortical desmoids are occasionally associated with mild pain, the majority of patients are asymptomatic, and the lesion is discovered as an incidental finding when radiography is performed for some other purpose [10,11].

Although detailed dissections implicated the extensor tendon of the adductor magnus muscle as the cause of the cortical irregularity in this region [2], in the earlier description by Kohler [9], the gastrocnemius and plantaris tendons were believed to be involved. Given the separate and distinct actions of the two muscles [12], it is not surprising that cortical stress injuries could involve either muscle insertion separately. Differentiation between stress injuries caused by the adductor magnus muscle and the medial head of the gastrocnemius is probably not possible using conventional radiography, inasmuch as the adductor tubercle and the insertion site of the medial head of the gastrocnemius tendon lie immediately adjacent to each other [12]. Such distinction is easily demonstrable by CT since the muscles can be individually identified. The adductor tubercle lies at the extreme posteromedial aspect of the distal femoral metaphysis, while the insertion of the medial head of the gastrocnemius muscle is more lateral (Fig. 2D).

In the majority of cases, plain radiographs are diagnostic of cortical desmoid. CT can be useful in equivocal or atypical cases.

\section{References}

1. Allen DH (1953) A variation of diaphyseal development which simulates the roentgen appearance of primary neoplasms of bone. AJR 69:940

2. Barnes GR, Gwinn JL (1974) Distal irregularities of the femur simlating malignancy. AJR 122:180

3. Brower AC, Culver JE, Keats TE (1971) Histological nature of the cortical irregularity of the medial posterior distal femoral metaphysis in children. Radiology 99:389

4. Bufkin WJ (1971) The avulsive cortical irregularity. AJR 112:487

5. Dunham WK, Marcus NW, Enneking WF, Haun C (1980) Developmental defects of the distal femoral metaphysis. $J$ Bone Joint Surg [Am] 62:801

6. Johnson LC, Genner BA III, Engh CA, Brown RH (1968) Cortical desmoids (Abstr). In: Proceedings of the American Academy of Orthopedic Surgeons. J Bone Joint Surg [Am] $50: 828$

7. Kimmelstiel P, Rapp I (1951) Cortical defect due to periosteal desmoids. Bull Hosp Joint Dis 12:286

8. Kirkpatrick JA, Wilkinson RH (1978) Case report 52. Skeletal Radiol 2:189

9. Kohler A (1928) Roentgenology: The borderlands of the normal and early pathological in the skiagram, 5th edn. William Wood, New York, p 164

10. Resnick D, Niwayama G (1981) Diagnosis of bone and joint disorders with an emphasis on articular abnormalities, Vol 3. Saunders, Philadelphia p 2663

11. Simon H (1968) Medial distal metaphyseal femoral irregularity in children. Radiology 90:258

12. Williams PL, Warwick R (eds) (1980) Gray's anatomy. 36th edn. Churchill Livingston, Edinburgh, pp 394, 607-8 